\title{
Prevalencia de tumor venéreo transmisible en perros callejeros de la ciudad de Mérida, Yucatán, México.
}

\section{Comunicación Breve}

Antonio Ortega-Pacheco, Martín Acevedo-Arcique, Carlos Sauri-Arceo, Manuel Bolio-González, Eduardo Gutiérrez-Blanco.

Departamento de Medicina Interna y Cirugía, Facultad de Medicina Veterinaria y Zootecnia, Universidad Autónoma de Yucatán, Mérida, Yucatán, México.

\section{RESUMEN.}

Introducción. El tumor venéreo transmisible (TVT) es una neoplasia específica del perro que ocurre generalmente en la mucosa de los genitales. Éste es transmitido principalmente durante el coito y se encuentra ampliamente distribuido en el ámbito mundial, donde los perros callejeros tienen un papel importante en la diseminación. El objetivo de este trabajo fue el de determinar la prevalencia de TVT en perros callejeros capturados y concentrados en la perrera municipal de la ciudad de Mérida Yucatán, México y determinar su frecuencia de acuerdo al sexo y edad de los animales.

Materiales y métodos. Se revisaron 303 perros capturados después de ser sacrificados en la perrera municipal de Mérida, en busca de masas sugestivas a TVT. Se realizaron frotis de las masas sospechosas y se evaluaron microscópicamente para determinar su origen. Se determino la prevalencia de esta patología y su frecuencia de acuerdo al sexo y la edad.

Resultados. Se encontró una prevalencia del $2.64 \%$ $(\mathrm{n}=8)$ la cual fue ligeramente mayor en hembras (3.03\%) que en los machos (2.18\%); ésta se observó en animales de 3 años y mayores 4 años de edad.

Discusión. Se encontró una baja prevalencia de esta neoplasia en los perros evaluados probablemente por ser poco diseminada en la población canina callejera de la ciudad o por regresión espontánea. El sexo no parece ser un factor importante en la aparición de esta patología y se observa con mayor frecuencia en animales adultos y viejos por estar más tiempo expuestos al riesgo. (Rev Biomed 2003; 14:83-87)

Palabras clave: Tumor venéreo transmisible, perros callejeros.

\section{SUMMARY.}

Prevalence of transmissible venereal tumor of stray dogs in Merida Yucatan Mexico.

Introduction. Transmissible venereal tumor (TVT) is a specific neoplasia in dogs that generally occurs in the mucosa of the genitalia. It is transmitted during

Solicitud de sobretiros: MVSc Antonio Ortega-Pacheco, Departamento de Medicina Interna y Cirugía. Facultad de Medicina Veterinaria y Zootecnia, Universidad Autónoma de Yucatán, Km. 15.5 Carr. Mérida-Xmatkuil, Apdo. Postal 4-116 Itzimná, C.P. 97100, Mérida, Yucatán, México. Tel (9) 942-32-00 Fax: (9) 942-32-05 E-mail: opacheco@tunku.uady.mx Recibido el 12/Abril/2002. Aceptado para publicación el 12/Julio/2002. 


\section{A Ortega-Pacheco, M Acevedo-Arcique, C Sauri-Arceo y col.}

coitus and is distributed world wide; stray dogs play an important role in the dissemination. The objective of this work was determine the prevalence of TVT in stray dogs captured at the municipal pound of Mérida and determine its frequency according to sex and age of dogs.

Material and Methods. A visual examination of 303 captured dogs was made after its sacrifice at the municipal pound of Mérida searching for neoplasias in the genitals. A frotis of the suspicious structures was made and were microscopically evaluated to determine its origin. Prevalence of the pathology and its frequency was determined according to sex and age of the animals.

Results. A prevalence of TVT was $2.64 \%(\mathrm{n}=8)$ and was slightly larger in females (3.03\%) than in males $(2.18 \%)$; it was observed in animals 3 to ${ }^{3} 4$ years old.

Discussion. A low prevalence was probably found because of its slow dissemination among stray dogs or due to spontaneous regression. The sex of the animals does not seem to be an important factor in the dissemination of this pathology; adult to old animals seem to be more predisposed to acquire this pathology for being at risk longer.

(Rev Biomed 2003; 14:83-87)

Key words: Transmisible venereal tumor, stray dogs.

\section{INTRODUCCIÓN.}

El tumor venéreo transmisible (TVT) conocido también como sarcoma infeccioso, linfosarcoma venéreo, granuloma venéreo, condiloma canino y tumor transmisible de células reticulares entre otros, es una neoplasia específica de los perros y uno de los más frecuentes en esta especie (1). Su apariencia puede ser desde un pequeño nódulo de $5 \mathrm{~mm}$ hasta una masa en forma de coliflor de $10 \mathrm{~cm}$ o mayor (2). La transmisión es un ejemplo típico de aloinjerto natural de células exfoliadas, entre un individuo infectado y sembradas en uno sano. El TVT se encuentra en la mucosa del tracto reproductivo de la hembra (generalmente en vulva y vestíbulo vaginal) y en el pene en el caso de los machos $(2,3)$. Sin embargo, se ha diagnosticado también en zonas extragenitales como la piel, cara, vías nasales, cavidad bucal e interior y contorno de los ojos (24); también se ha encontrado en el bazo, hígado, cerebro y pulmones $(1,3)$, lo que indica su capacidad de metástasis. El TVT se propaga y se mantiene en la población de perros a través del coito, lameduras o mordeduras y son los perros callejeros los que sirven como reservorios de esta enfermedad $(2,5)$. $\mathrm{Su}$ frecuencia se reporta entre un 16\% (6) hasta el $34 \%$ (7). El contacto sin supervisión entre perros mantenidos en zonas con alta densidad de población de perros callejeros son de los principales factores de riesgo que pueden favorecer el contagio (7). En Mérida existe una gran cantidad de perros callejeros que en poca proporción son capturados y remitidos a la perrera municipal. Estos perros son portadores de diversas enfermedades, muchas de ellas zoonóticas (8). Entre las enfermedades reproductivas el TVT es de las más comunes que pueden ser transmitidas a perros cuyos propietarios los pasean por los parques públicos o que tienen libre acceso a las calles.

El objetivo del presente trabajo fue determinar la prevalencia de TVT en perros callejeros capturados y concentrados en la perrera municipal de la ciudad de Mérida, Yucatán, México y establecer si el sexo y la edad tienen un papel importante en la aparición de esta patología.

\section{MATERIAL Y MÉTODOS.}

Localización. Este estudio se realizó en la perrera municipal de la ciudad de Mérida, Yucatán, México en el lapso de julio a septiembre de 2001. Los perros eran capturados de las calles y sacrificados 3 días después de su arribo si no eran reclamados. Anualmente son sacrificados entre 2,500 y 3,000 perros de distintas razas, sexos y edades.

Animales. Se evaluaron 303 perros callejeros capturados y sacrificados en la perrera municipal de Mérida, considerando dentro del muestreo únicamente a los que fueron mayores de un año de edad; de manera que se consideró que éstos eran púberes $\mathrm{y}$, por lo tanto, con riesgo de infectarse por

\section{Revista Biomédica}


Tumor venéreo transmisible en perros.

contacto sexual.

Tamaño de muestra. Esta se determinó utilizando el programa Win Episcope 2, basado en la fórmula para determinar la prevalencia (9). Se evaluaron 303 perros, considerando un nivel de confianza del $95 \%$ y un error del $5 \%$, con una prevalencia esperada del $34 \%$ (7).

Determinación del sexo y edad. Después de sacrificados los animales, se determinaron: el sexo, por observación visual de los genitales externos, y la edad según la metodología descrita (10) para la dentición del animal:

1 año. Incisivos superiores e inferiores sin desgaste, presentando la corona y las dos cúspides completas.

2 años. La corona de los incisivos inferiores comienzan a desgastarse ligeramente.

3 años. La corona de los incisivos inferiores se encuentran completamente desgastadas y se empiezan a desgastar las cúspides.

4 años. La corona de los incisivos superiores comienza a desgastarse y en los inferiores se encuentran totalmente desgastados la corona y cúspides.

5 años. La corona de los incisivos superiores se encuentra totalmente desgastada y comienzan a desgastarse las cúspides; los incisivos inferiores se encuentran totalmente desgastados en la corona y cúspide.

6 años. Los incisivos superiores e inferiores se encuentran totalmente erosionados

7 años Las piezas dentarias comienzan a ladearse hacia fuera, hay acumulación de sarro que da una coloración amarillenta y pueden faltar piezas dentarias.

Toma de muestras y observaciones. Se realizó una inspección visual y física de los perros sacrificados, con especial interés en las áreas genitales en busca de características macroscópicas del tumor $(1,2,11,12)$. Una vez detectada la neoplasia, se realizó una impronta, la cual se tiñó inmediatamente con DiffQuik y se le dio lectura para determinar si se trataba o no de TVT en base a las características microscópicas del tumor $(2,11,13,14)$.
Análisis estadístico. Se determinó la prevalencia de TVT y su frecuencia de presentación por sexo y edad en la población de perros callejeros capturados por la perrera municipal.

\section{RESULTADOS.}

Se encontró una prevalencia de TVT del $2.64 \%$ la cual fue ligeramente mayor en hembras que en los machos (cuadro 1).

La frecuencia de presentación de TVT según el sexo y la edad se observa en el cuadro 2. De los 8 casos, 7 se presentaron en perros (4 hembras y 3 machos) de 3 años de edad y uno en una hembra de 5 años por lo que se incluyó en el rubro de 34 años. Todos los casos de TVT detectados en el macho se encontraron en el pene, mientras que en el caso de las hembras, se detectó su presencia en la vulva y/o vagina.

\section{DISCUSIÓN.}

Los resultados de este trabajo señalan que el TVT es una enfermedad presente en los perros callejeros de la ciudad de Mérida. Los perros callejeros, como ha sido señalado (5), sirven de reservorios para esta enfermedad y representan un riesgo para la población de perros sanos callejeros o aquellos que tienen libre acceso a la calle, puesto que al tener contacto sexual o incluso al olfatear los genitales de un perro infectado pueden contraer la enfermedad. Al ser el TVT de transmisión sexual, su propagación podría ser más rápida en Mérida ya que se ha observado una tendencia de las perras a manifestar celos en épocas particulares del año (15), de tal manera que en grupos sexualmente activos, la presencia de un animal padeciendo de TVT, sobre todo si se trata de una hembra en celo, podría ser

\section{Cuadro 1}

Prevalencia de TVT en perros callejeros capturados en la perrera municipal de la ciudad de Mérida según el sexo.

\begin{tabular}{lccc}
\hline SEXO & n & CASOS POSITIVOS & \% \\
\hline Macho & 138 & 3 & 2.18 \\
Hembra & 165 & 5 & 3.03 \\
\hline Total & 303 & 8 & 2.64 \\
\hline
\end{tabular}

Vol.14/No.2/Abril-Junio, 2003 
A Ortega-Pacheco, M Acevedo-Arcique, C Sauri-Arceo y col.

Cuadro 2

Prevalencia de TVT en perros callejeros capturados en la perrera municipal de la ciudad de Mérida según su edad y sexo.

\begin{tabular}{cccccc}
\hline EDAD (años) & HEMBRAS (n) & HEMBRAS + & MACHOS (n) & MACHOS + TOTAL \\
\hline 1 & 25 & 0 & 23 & 0 & 0 \\
2 & 28 & 0 & 25 & 3 & 0 \\
3 & 93 & 4 & 17 & 0 & 1 \\
\hline 4 & 19 & 1 & 138 & 3 \\
\hline
\end{tabular}

suficiente para propagar la infección al resto del grupo. Existe evidencia de que esta patología puede presentar un patrón estacional (16), de manera que se detectan más casos en primavera y verano que en otras épocas del año; lo anterior coincide con las tendencias de encontrar más perras en estro en Mérida.

La prevalencia encontrada en este trabajo está por debajo de otros estudios en donde se señalan prevalencias de $6.8 \%$ (17), $16 \%$ (6) y $34 \%$ (7). Uno de los principales factores de riesgo reportados (7) es la alta densidad de perros callejeros, lo cual no ha sido cuantificado en la ciudad de Mérida, Yucatán. Además, estos estudios fueron realizados en zonas rurales donde existe poca supervisión de perros los cuales en su mayoría son callejeros. Por ello representan focos importantes sobre todo en poblados cercanos a la ciudad, ya que éstos pueden incorporarse con facilidad a la población canina urbana. Por otro lado, el TVT puede en un porcentaje sufrir la regresión espontánea completa, incluso antes de haber cursado con cuadros clínicos aparentes (14). Esta patología es atendida con cierta regularidad en las clínicas particulares de la ciudad de Mérida, lo cual en su mayoría está asociado a que la mascota tuvo acceso a la calle sin supervisión o se realizó la monta en la cual alguno de los animales estuvo en contacto con algún otro perro callejero (Reportes no publicados de la Asociación de Médicos Veterinarios Especialistas en Pequeñas Especies de Yucatán y el Colegio de Médicos Veterinarios Especialistas en Pequeñas Especies de Yucatán).

Este tumor por ser un aloinjerto natural, sus células pueden incluso implantarse y proliferar en otros sitios como la nariz, ano, boca y piel, entre otros
$(1,3,18,19)$. Sin embargo, en este trabajo no se observó TVT extragenital probablemente por la baja prevalencia encontrada.

El sexo del animal no pareció predisponer la aparición de esta patología, pues se presentó indistintamente en machos como en hembras. Otros trabajos señalan que de 28 casos detectados de TVT, 15 fueron machos y 13 hembras (6); contrariamente, en otro estudio se señala que el $68.5 \%$ de animales con esta patología fueron hembras y el $31.5 \%$ fueron machos (20). Aunque en este trabajo se observó una tendencia de las hembras a padecer con mayor frecuencia TVT, el hallazgo no parece tener mayor relevancia.

En cuanto a la edad de los perros, ésta representa un factor de riesgo sobre todo si son callejeros desde que el animal inicia la pubertad y tiene contacto con hembras en estro, o incluso con machos dominantes quienes por su comportamiento pueden montar a otros machos $(3,21)$. El TVT aparece desde el año de edad con un rango de 1-3 años (6) o de 15 años de edad (14). En este trabajo, las edades en las cuales se encontró TVT fue de 3 años y mayores de 4 años de edad, probablemente porque la mayoría de los perros capturados se encontraron dentro de estas edades. Además, el tiempo de riesgo se incrementa mientras mayor sea la edad del animal y se aparee. Se concluye que el TVT es una patología presente en la población canina callejera de la ciudad de Mérida, aunque con una baja prevalencia; el sexo no parece ser un factor que predisponga la aparición de esta enfermedad, la cual es más probable de encontrarse en animales adultos por estar mayor tiempo en riesgo.

\section{Revista Biomédica}




\section{Tumor venéreo transmisible en perros.}

\section{REFERENCIAS.}

1.- Moulton JE. Tumors in Domestic Animals. California USA: University of California Press; 1990.

2.- Rogers KS. Transmissible venereal tumor. Compendium on Continuing Education 1997; 19: 273-275.

3.- Cohen D. The mechanisms of transmission of the transmissible venereal tumor of the dog. Transplantation 1974; 17:8-11.

4.- Boscos CM, Ververidis HN, Tondis DK, Stamou AI, Samartzi FC. Ocular involvement of transmissible venereal tumor in a dog. Veterinary Ophthalmology 1998; 1: 163-170.

5.- Brown NO. Transmissible venereal tumor in the dog. California Veterinary 1981; 3:6.

6.- Batamuzi EK, Bittegeko SBP. Canine transmissible tumor in Morongo, Tanzania. Zimbabwe. Veterinary Journal 1990; 21: $152-154$.

7.- Batamuzi EK, Kassuku AA, Agger JF. Risk factors associated with canine transmissible venereal tumor in Tanzania. Preventive Veterinary Medicine 1992; 13: 13-17.

8.- Faulkner LC. Dimensions of the pet population problem. JAVMA 1975; 166 : 477-478.

9.- Thusfield M. Veterinary Epidemiology. $2^{\text {nd }}$ Edition, Edinburgh: Blackwell Science; 1995. p. 177-190.

10.- Payró LJ. El Perro y su Mundo, Tratado de Zootecnia Canina. Loera Chávez Hnos., México, 1981. p. 55-58.

11.- Wright JP, Parry WB. Cytology of the canine reproductive system. Veterinary Clinics of North America: Small Animal Practice 1989; 19: 851-873.

12.- Morgan R. Clínica de Pequeños Animales. $3^{\mathrm{a}}$ Edición, Editorial Harcourt Brace Saunders, Madrid España 1999. pp. 612-614.

13.- Fernández O, Gómez L, Gázquez A, Durán M. El diagnóstico citológico en las neoplasias cutáneas caninas. Consulta de Difusión Veterinaria, 1999; 7:91-103.

14.- Feldman EC, Nelson RW. Endocrinología y Reproducción en Perros y Gatos. $2^{\mathrm{a}}$. Edición, México: McGraw-Hill Interamericana. 2000. p. 725-728.

15.- Ortega PA, Rodríguez BJC, Leal OJ. Actividad estral de perras callejeras en la ciudad de Mérida Yucatán y su relación con edad, talla y condición corporal. Rev Biomed 2000; 11:107111.

16.- Gandotra VK, Chauhan FS, Sharma RD. Occurrence of canine transmissible venereal tumor and evaluation of two treatments. Indian Veterinary Journal 1993; 70: 854-857.

17.- Rautenbach GH, Boomker J, De Villiers LL. A descriptive study of the canine population in a rural town in southern Africa. Journal of the Sudafrican Veterinary Association 1991; 62: $158-162$.

18.- Miller WW, Albert RA, Boosinger TR. Ocular metastasis of a transmissible venereal tumor. Canine practice 1990; 15: 19-21.

19.- Bravo J, De Buen N, Colin R. Diagnóstico citológico del tumor venéreo transmisible extragenital. Informe de un caso. AMMVEPE 1995; 33: 396-398.

20.- Gandotra VK, Prabhakar S, Singla VK, Chauhan FS, Sharma RD. Incidence of physiopathological reproductive problems in canines. Indian Veterinary Journal 1993; 70: 467.

21.- Pereira JT, Ramadinha RHR. Prevalencia do tumor venereo transmissiver canino quanto a sexo, ruade e raca. Arquivos da Universidade Federal Rural do Rio de Janeiro 1991; 14: 143-146. 